\title{
Diagnostic accuracy of a novel point-of-care urine lipoarabinomannan assay for the detection of tuberculosis among adult outpatients in Zambia: a prospective cross- sectional study
}

\author{
Monde Muyoyeta $\mathbb{1}^{1,4}$, Andrew D. Kerkhoff $\mathbb{C}^{2,4}$, Lophina Chilukutu ${ }^{1}$, Emmanuel Moreau ${ }^{3}$, \\ Samuel G. Schumacher $\mathbb{1}^{3}$ and Morten Ruhwald ${ }^{3}$
}

${ }^{1}$ Centre for Infectious Diseases Research in Zambia, Lusaka, Zambia. ${ }^{2}$ Division of HIV, Infectious Diseases and Global Medicine, Zuckerberg San Francisco General Hospital and Trauma Center, University of California San Francisco, San Francisco, CA, USA. ${ }^{3}$ Foundation for Innovative New Diagnostics (FIND), Geneva, Switzerland. ${ }^{4}$ These authors contributed equally to this work.

Corresponding author: Monde Muyoyeta (Mondemuy@gmail.com)

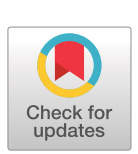

Copyright $\odot$ The authors 2021

This version is distributed under the terms of the Creative Commons Attribution Licence 4.0.

This article has supplementary material available from erj.ersjournals.com

Received: 29 Oct 2020 Accepted: 5 April 2021

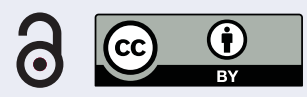

Shareable abstract (@ERSpublications)

In a prospective study, the FujiLAM urine-based point-of-care test demonstrated high sensitivity of $\geqslant 75 \%$ for the detection of TB among both HIV-positive and HIV-negative ambulatory adults, and also had good specificity. https://bit.ly/328jrod

Cite this article as: Muyoyeta M, Kerkhoff AD, Chilukutu L, et al. Diagnostic accuracy of a novel pointof-care urine lipoarabinomannan assay for the detection of tuberculosis among adult outpatients in Zambia: a prospective cross-sectional study. Eur Respir J 2021; 58: 2003999 [DOI: 10.1183/ 13993003.03999-2020].

\section{Abstract}

Background A novel, rapid, point-of-care urine-based lipoarabinomannan assay (Fujifilm SILVAMP TB LAM ("FujiLAM")) has previously demonstrated substantially higher sensitivity for tuberculosis (TB) compared with the commercially available Determine TB LAM assay using biobanked specimens. However, FujiLAM has not been prospectively evaluated using fresh urine specimens. Therefore, we determined the diagnostic accuracy of FujiLAM among HIV-positive and HIV-negative outpatients with presumptive TB in Zambia.

Methods Adult ( $\geqslant 18$ years old) presumptive TB patients presenting to two outpatient public health facilities in Lusaka were included. All patients submitted sputa samples for smear microscopy, Xpert MTB/RIF and mycobacterial culture, and urine samples for the FujiLAM assay. Microbiologically confirmed TB was defined by the detection of Mycobacterium tuberculosis in sputum using culture; this served as the reference standard to assess the diagnostic accuracy of FujiLAM.

Results 151 adults with paired sputum microbiological tests and urine FujiLAM results were included; 45\% were HIV-positive. Overall, 34 out of 151 (23\%) patients had culture-confirmed pulmonary TB. The overall sensitivity and specificity of FujiLAM was 77\% (95\% CI 59-89\%) and 92\% (95\% CI 86-96\%), respectively. FujiLAM’s sensitivity among HIV-positive patients was 75\% (95\% CI 43-95\%) compared with 75\% (95\% CI 51-91\%) among HIV-negative patients. The sensitivity of FujiLAM in patients with smear-positive, confirmed pulmonary TB was 87\% (95\% CI 60-98\%) compared with 68\% (95\% CI 43-87\%) among patients with smear-negative, confirmed pulmonary TB.

Conclusions FujiLAM demonstrated high sensitivity for the detection of TB among both HIV-positive and HIV-negative adults, and also demonstrated good specificity despite the lack of systematic extrapulmonary sampling to inform a comprehensive microbiological reference standard.

\section{Introduction}

The development of new, highly sensitive, point-of-care tuberculosis (TB) diagnostic tests that are not dependent on a sputum sample are key to meeting the End TB Strategy targets [1]. These diagnostic tests should be affordable and easy to use, to facilitate scale-up in high-burden, resource-constrained settings where there is the most need. In 2019, the World Health Organization (WHO) strengthened and expanded 
upon its recommendations on the use of the Determine TB LAM ("LF-LAM") test (Abbott Diagnostics, Lake Bluff, IL, USA), a rapid, urine-based, point-of-care assay, to diagnose TB among people living with HIV (PLHIV) [2].

The LF-LAM test is based on the detection of mycobacterial lipoarabinomannan (LAM). LAM is a cell wall byproduct of replicating mycobacterial bacilli that can be detected in the blood, sputum and urine of persons with TB, although development of urine-based LAM assays is attractive due to ease of specimen collection. LAM is a heterogenous molecule with four structural domains, including a domain highly preserved across mycobacterial species as well as variable domains that may serve as target epitopes for the development of TB diagnostic tests [3, 4]. Despite LF-LAM's low cost, ease of use and demonstrated mortality benefit, its uptake has been slow [5]. This is largely due to its low sensitivity in most patient groups, with the exception being individuals with advanced immune deficiency [6].

The Fujifilm SILVAMP TB LAM ("FujiLAM”) (Fujifilm, Tokyo, Japan) is a next-generation, rapid, urine-based LAM test that has been demonstrated to have two-fold higher sensitivity than LF-LAM in PLHIV (71\% versus 35\%) using biobanked specimens and it also retained useful sensitivity at higher CD4 count levels as well as among HIV-negative individuals [7, 8]. Compared with LF-LAM, which is a lateral flow assay that uses polyclonal antibodies, FujiLAM utilises high-affinity monoclonal antibodies directed towards largely $M$. tuberculosis-specific LAM epitopes and adds a silver amplification step; this facilitates detection of LAM concentrations 30 times lower than can be detected using the LF-LAM assay, while also providing improved analytic specificity [9]. However, the diagnostic accuracy of the FujiLAM assay has not previously been prospectively evaluated on fresh urine specimens and to date the available data among people without HIV are very limited [8]. We report and compare the diagnostic accuracy of FujiLAM among HIV-positive and HIV-negative presumptive TB patients in an outpatient setting, using fresh specimens.

\section{Methods}

\section{Study design and participants}

This diagnostic evaluation study was nested within a larger, ongoing TB diagnostic study. Adults ( $\geqslant 18$ years old) attending Kanyama First Level Hospital and Chainda Health Centre (Lusaka, Zambia) between January 2019 and July 2019 were screened for the presence of any TB symptoms. Kanyama First Level Hospital serves a large densely populated peri-urban township with a high prevalence of HIV and incidence of TB; TB notification rates typically exceed $>2000$ per 100000 in this setting. Adult presumptive TB patients without a current TB diagnosis were eligible for study inclusion and those who agreed to participate were consecutively enrolled. Patients were defined as having "presumptive TB" if any of the following symptoms were present (regardless of duration): cough, fever, night sweats or unintentional weight loss, or abnormal chest radiography (regardless of symptoms).

The study had ethical approval from the Biomedical Research Ethics Committee of the University of Zambia (Lusaka, Zambia). All participants provided written informed consent in their primary language.

\section{Procedures and samples}

All patients completed a case record form that collected demographic details, presenting symptoms, past medical history, HIV status and physical examination findings. HIV status was by self-report; however, for those without a known or recently determined HIV status, they were offered opt-out HIV testing in accordance with local procedures. Participants were offered digital chest radiography (Odelca-DR; Delft Imaging Systems, 's-Hertogenbosch, The Netherlands) when available as part of the screening process. All patients submitted sputum and urine samples that were sent to an accredited laboratory for same-day processing. Sputum samples were processed for mycobacterial culture using the NALC-NaOH method: the pellet was inoculated on mycobacteria growth indicator tubes (MGIT; Becton Dickinson, Franklin Lakes, NJ, USA) and Löwenstein-Jensen medium (Becton Dickinson). The leftover pellet was used for Xpert MTB/RIF testing and concentrated acid-fast bacilli (AFB) Ziehl-Neelsen testing. Culture-positive tubes confirmed to contain AFB were tested with the MGIT TBc identification test (Becton Dickinson) to confirm the presence of $M$. tuberculosis [10]. The FujiLAM assay was performed on fresh urine specimens on the same day as sample collection according to the manufacturer's instructions [11]. CD4 cell counts were not systematically performed as part of this study.

\section{Data analysis and definitions}

Patient characteristics were compared according to HIV status using simple descriptive statistics. Proportions were compared using either Pearson's Chi-squared test or Fisher's exact test as appropriate, while the Wilcoxon rank-sum test was used to compare median values. The detection of $M$. tuberculosis in 
sputum using culture defined the microbiological reference standard for diagnostic accuracy calculations. The sensitivity, specificity, positive predictive value (PPV) and negative predictive value (NPV) with corresponding 95\% confidence intervals were determined for sputum smear microscopy, Xpert MTB/RIF and FujiLAM. The sensitivities of microbiological TB investigations for culture-confirmed TB were compared and visualised using proportional Venn diagrams. To better understand the association between FujiLAM positivity and mycobacterial burden, we determined the sensitivity of FujiLAM classified according to sputum Xpert MTB/RIF semiquantitative result (e.g. very low, low, medium or high).

\section{Results}

Of 183 adult presumptive TB patients recruited, 157 were enrolled; 151 had complete microbiological results and were included in the analysis (figure 1). 89 (59\%) patients were male, the median (interquartile range) age was 37 (28-43) years, 68 (46\%) were HIV-positive and 32 (21\%) had a prior history of TB. Compared with HIV-negative patients, HIV-positive patients were slightly older, less likely to report cough or fevers and were substantially more likely to report a prior history of active TB disease (table 1). Overall, 34 out of 151 (23\% (95\% CI 16-30\%)) patients had culture-confirmed pulmonary TB; the prevalence of TB was 18\% (95\% CI 9-29\%) among HIV-positive patients and 25\% (95\% CI 16-36\%) among HIV-negative patients.

\section{Diagnostic accuracy of FujiLAM}

FujiLAM had an overall sensitivity and specificity of 77\% (95\% CI 59-89\%) and 92\% (95\% CI 86-96\%), respectively (figure 2). Among HIV-positive individuals, the sensitivity and specificity of FujiLAM was 75\% (95\% CI 43-95\%) and 89\% (95\% CI 78-96\%), respectively, while among HIV-negative individuals the sensitivity and specificity was 75\% (95\% CI 51-91\%) and 95\% (95\% CI 86-99\%), respectively. FujiLAM's sensitivity among those with smear-positive TB was 87\% (95\% CI 60-98\%); among smear-negative individuals, the sensitivity and specificity of FujiLAM was 68\% (95\% CI 43-87\%) and 92\% (95\% CI 86-96\%), respectively (supplementary table S1). The characteristics of the nine patients with a "false-positive" FujiLAM result are shown in supplementary table S2; six patients were HIV-positive, three had a prior history of TB, eight had current cough, six had recent weight loss, five had current night sweats and four had current fever; one patient had a positive Xpert MTB/RIF result.

The overall PPV for FujiLAM was 74\% (95\% CI 57-88\%); PPVs among HIV-infected and HIV-negative patients were 60\% (95\% CI 32-84\%) and 83\% (95\% CI 59-96\%), respectively (supplementary table S3). The overall NPV for FujiLAM was 93\% (95\% CI 87-97\%); NPVs among HIV-infected and HIV-negative patients were 94\% (95\% CI 84-99\%) and 92\% (95\% CI 82-97\%), respectively.

\section{Comparative diagnostic sensitivity of tuberculosis tests}

The comparative diagnostic sensitivity of different TB tests among those with culture-confirmed, pulmonary TB is shown in figure 3. FujiLAM demonstrated higher diagnostic sensitivity compared with sputum smear microscopy (77\% versus 44\%) (supplementary table S1). While the addition of FujiLAM to sputum smear microscopy increased sensitivity by $38 \%$ ( $n=13$ cases), the additive sensitivity of sputum smear microscopy following FujiLAM was only 6\% (n=2 cases). All TB cases were detectable using Xpert MTB/RIF.

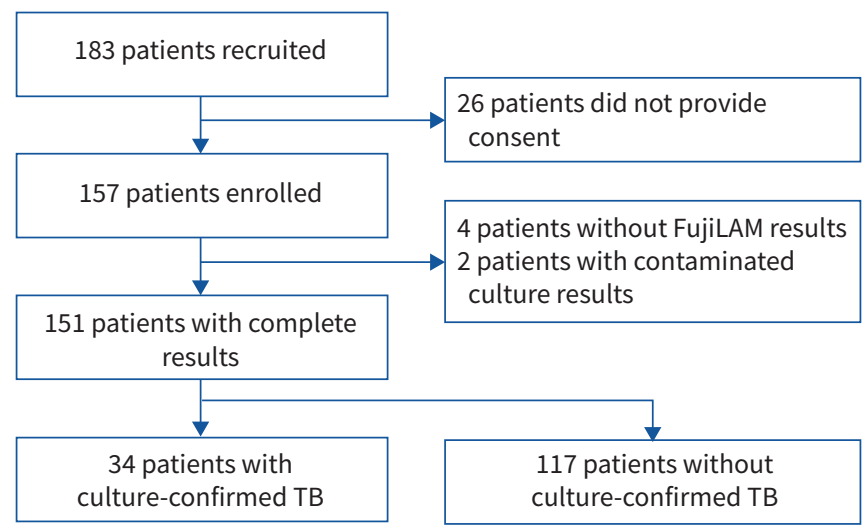

FIGURE 1 Study flow diagram. TB: tuberculosis. 
TABLE 1 Patient characteristics according to HIV status

\begin{tabular}{lccc} 
& All patients & HIV-positive patients & HIV-negative patients \\
\hline Patients & 151 & 68 & 81 \\
\hline Age years & $37(28-43)$ & $39(31-44)$ & $33(26-43)$ \\
Male & $89(58.9)$ & $37(54.4)$ & $50(61.7)$ \\
\hline Symptoms (any duration) & & & $70(86.4)$ \\
$\quad$ Cough & $123(81.5)$ & $51(75.0)$ & $50(61.7)$ \\
\hline Fevers & $81(53.6)$ & $29(42.7)$ & $58(71.6)$ \\
$\quad$ Weight loss & $109(72.2)$ & $49(72.1)$ & $61(75.3)$ \\
$\quad$ Night sweats & $106(70.2)$ & $44(64.7)$ & $11(13.6)$ \\
$\quad$ Haemoptysis & $22(14.6)$ & $10(14.7)$ & $80(98.8)$ \\
WHO symptom screen-positive & $145(96.0)$ & $63(92.7)$ & $5(6.2)$ \\
\hline New HIV diagnosis & $2(1.3)$ & $2(2.9)$ & $20(24.7)$ \\
\hline Previous history of TB & $32(21.2)$ & $27(39.7)$ & $12(17.7)$ \\
\hline Sputum culture TB-positive & $34(22.5)$ & & \\
\hline
\end{tabular}

Data are presented as $\mathrm{n}$, median (interquartile range) or $\mathrm{n}(\%)$. WHO: World Health Organization; TB: tuberculosis. ": two patients did not have HIV status results available.

\section{Relationship between FujiLAM and mycobacterial burden}

The relationship between FujiLAM positivity and Xpert MTB/RIF semiquantitative result among the 34 sputum culture-positive patients is shown in figure 4. Among HIV-positive patients, FujiLAM detected TB in all patients with low, medium or high sputum Xpert positive results, but missed TB in the three patients with a very low Xpert positive result, suggesting a relationship between FujiLAM and mycobacterial burden in HIV-positive patients. Among HIV-negative patients, there was no clear association between FujiLAM and the Xpert MTB/RIF semiquantitative result (figure 4).

\section{Discussion}

In this first prospective study to evaluate the diagnostic accuracy of FujiLAM, we found that it had high sensitivity for the detection of TB among ambulatory adults with presumptive TB in Zambia. FujiLAM also demonstrated good specificity despite the lack of a comprehensive reference standard. Notably, FujiLAM's diagnostic accuracy did not substantially differ between HIV-positive and HIV-negative

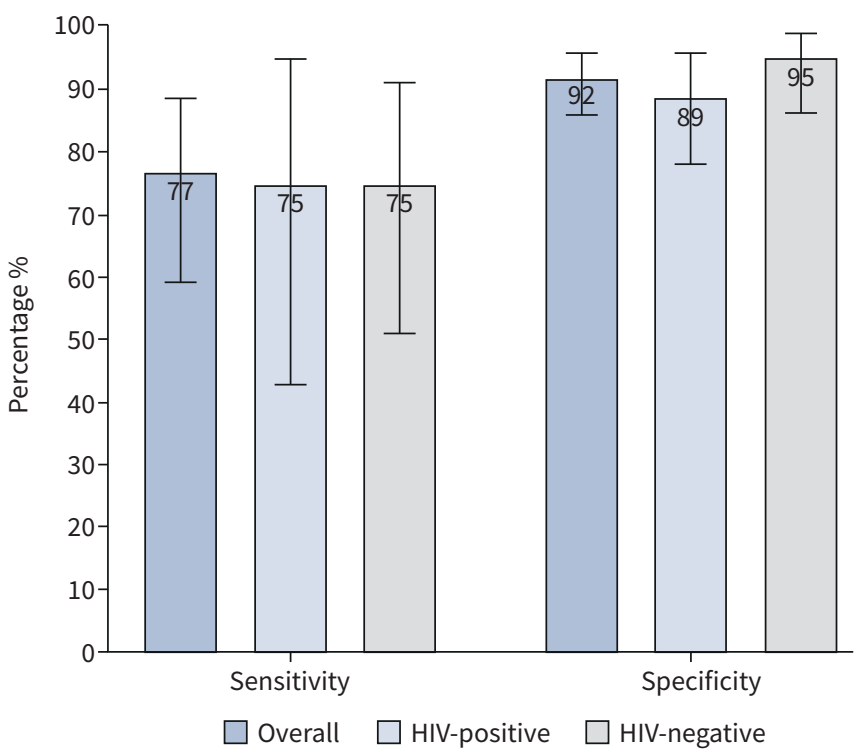

FIGURE 2 Diagnostic sensitivity and specificity of FujiLAM overall ( $n=151$ patients) and according to HIV status (HIV-positive $n=68$ patients and HIV-negative $n=81$ patients). Two patients did not have HIV status results available. Error bars indicate $95 \% \mathrm{Cl}$. 


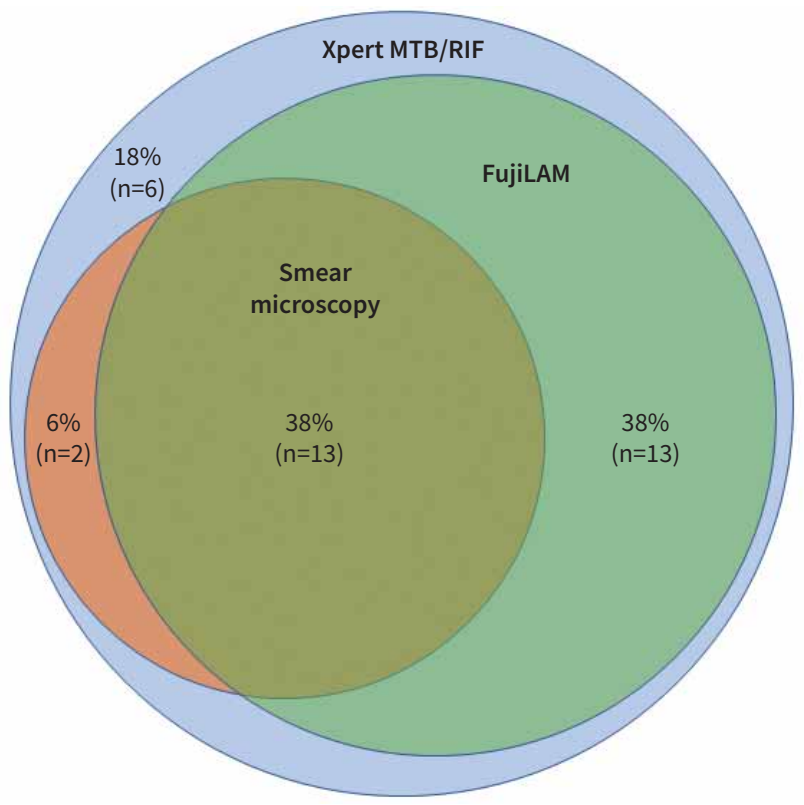

FIGURE 3 Proportional Venn diagram showing the diagnostic sensitivity and number of culture-confirmed tuberculosis cases detected using different assays ( $n=34$ patients).

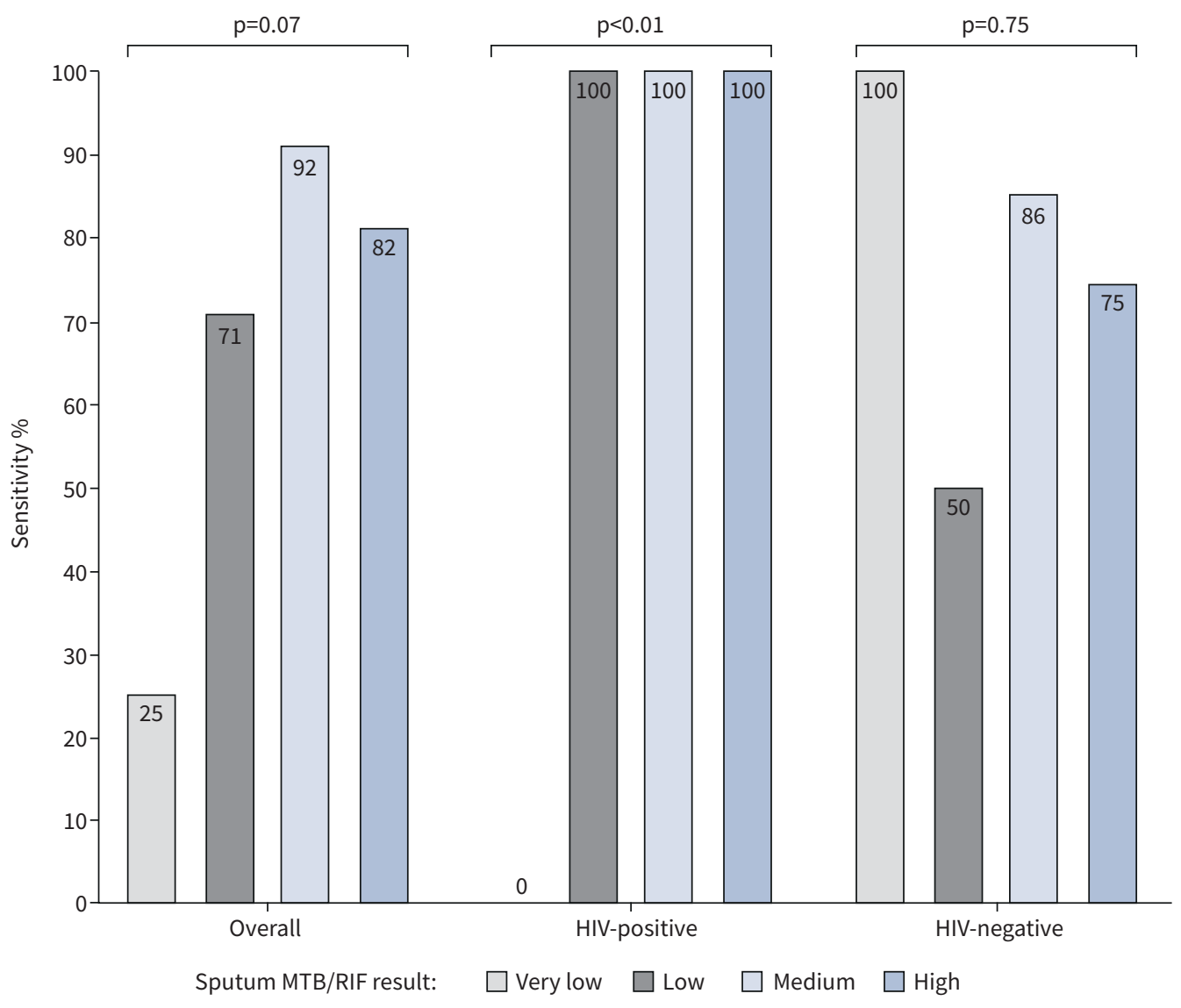

FIGURE 4 Association between FujiLAM sensitivity and Xpert MTB/RIF semiquantitative result according to HIV status among culture-confirmed tuberculosis cases (overall $n=34$ patients: HIV-positive $n=12$ patients and HIV-negative $n=20$ patients). 
patients; however, our sample size was limited and additional, larger studies are needed to confirm these findings.

The diagnostic sensitivity of FujiLAM among HIV-positive patients in our study was consistent with prior evaluations. While our diagnostic accuracy results are informed by a relatively small number of ambulatory HIV-positive patients, it is encouraging that our results (sensitivity 75\% (95\% CI 43-95\%)) are similar to previous evaluations conducted using biobanked specimens from three outpatient cohorts (pooled sensitivity 71\% (95\% CI 45-90\%)) and three inpatient cohorts (pooled sensitivity 70\% (95\% CI 53-83\%)) [7]. Among HIV-negative patients in our study, FujiiLAM's sensitivity (75\% (95\% CI 51-91\%)) was higher than prior pooled estimates using biobanked specimens from three outpatient cohorts (53\% (95\% CI 44-62\%)) [8]. While this may in part reflect the limited numbers of patients included and there may be no difference (overlapping confidence intervals), it may also reflect differences in the severity of TB disease and total mycobacterial burden among participants. FujiLAM's sensitivity appears to increase with greater mycobacterial burden [7, 8]; however, only 50\% of HIV-negative patients in our study had smear-positive disease compared with $68 \%$ of patients in the previous study [8]. Differences in FujiLAM's sensitivity may also be due to differences in fresh versus frozen urine specimens; however, we previously found that only minor improvements in sensitivity may be observed when using fresh specimens [12]. Further studies will be needed to clarify this finding.

We found that the sensitivity of FujiLAM far exceeded that of smear microscopy (77\% versus $44 \%$ ). FujiLAM was able to detect TB in the large majority of those with smear-positive disease (87\%) as well as the majority of those with smear-negative disease (64\%). Our results suggest that FujiLAM used in conjunction with smear microscopy would nearly double the sensitivity of smear microscopy alone, but that smear microscopy would add little incremental yield beyond FujiLAM. Unfortunately, we were not able to directly compare FujiiLAM's sensitivity to the commercially available LF-LAM assay. However, in evaluations using biobanked specimens, FujiLAM had 35-40\% greater pooled sensitivity among both HIV-positive (71\% versus 35\%) and HIV-negative patients (53\% versus $11 \%$ ) $[7,8]$.

FujiLAM's diagnostic sensitivity was less than that of Xpert MTB/RIF in this cohort, but comparable to that of Xpert Ultra among PLHIV with pulmonary TB in prior evaluations [13]. Roll-out of the Xpert platform in resource-constrained settings has faced challenges due to the need for a continuous power supply and the suboptimal laboratory conditions in primary healthcare settings that may result in prolonged down time [14]. Additionally, sputum Xpert may fail to detect TB among individuals with extrapulmonary and disseminated disease, a group in which FujiLAM appears to have high sensitivity but that we were unable to directly assess in the present study due to a lack of systematic extrapulmonary TB investigations [15]. Collectively, this suggests that FujiLAM could potentially be used as a first-line test to facilitate rapid TB diagnosis among presumptive TB patients, independent of HIV status, either as part of a parallel testing strategy with Xpert or in settings were Xpert remains unavailable.

Overall, FujiLAM had a good specificity (92\%) that was consistent with previously published assessments (93\%) [7, 8]. Reasons for potentially false-positive results need to be further explored in subsequent studies to clearly define patient populations among whom the test should be used to achieve sufficiently high specificity and avoid overtreatment. In the present study we did not systematically include extrapulmonary sampling to inform a comprehensive microbiological reference standard; this likely resulted in some misclassification, especially among HIV-positive patients as was suggested by the slightly lower specificity and PPV in this group compared with HIV-negative patients. For example, prior evaluations of FujiLAM using biobanked specimens have found its specificity to be up to $96 \%$ and $99 \%$ among HIV-positive and HIV-negative patients, respectively, when a composite reference standard including clinical diagnoses is utilised. Additionally, cross-reactivity with nontuberculous mycobacteria may have contributed to FujiLAM's reduced specificity in our study and should be specifically evaluated in future studies; however, preliminary evidence suggests that this is less problematic than for LF-LAM [16].

In conclusion, while larger prospective studies are required and are currently underway with the FujiLAM Prospective Evaluation Trial (ClinicalTrials.gov: NCT04089423), our results extend upon prior evaluations using biobanked specimens and suggest that the FujiLAM point-of-care test may have high sensitivity for the detection of TB among both HIV-positive and HIV-negative adults.

Acknowledgements: We would like to acknowledge Paul Somwe, Winnie Mwanza, Joel Bwalya and Paul Kaunda (Centre for Infectious Diseases Research in Zambia, Lusaka, Zambia) for their contributions to this study. We would also like to thank the Lusaka District Health Office Staff. 
Conflict of interest: M. Muyoyeta has nothing to disclose. A.D. Kerkhoff has nothing to disclose. L. Chilukutu has nothing to disclose. E. Moreau reports working for FIND. FIND is a non-for-profit foundation, whose mission is to find diagnostic solutions to overcome diseases of poverty in LMICs. It works closely with the private and public sectors and receives funding from some of its industry partners (no funding received from Cepheid for the development or the evaluation of Xpert). It has organisational firewalls to protect it against any undue influences in its work or the publication of its findings. All industry partnerships are subject to review by an independent scientific advisory committee or another independent review body, based on due diligence, TTPs and public sector requirements. FIND catalyses product development, leads evaluations, takes positions and accelerates access to tools identified as serving its mission. It provides indirect support to industry (e.g. access to open specimen banks, a clinical trial platform, technical support, expertise, laboratory capacity strengthening in LMICs) to facilitate the development and use of products in these areas. FIND also supports the evaluation of publicly prioritised TB assays and the implementation of WHO-approved (guidance and $\mathrm{PQ}$ ) assays using donor grants. In order to carry out test evaluations, FIND has product evaluation agreements with several private sector companies for TB and other diseases, which strictly define its independence and neutrality vis-à-vis the companies whose products get evaluated and describes roles and responsibilities. S.G. Schumacher reports working for FIND. FIND is a non-for-profit foundation, whose mission is to find diagnostic solutions to overcome diseases of poverty in LMICS. It works closely with the private and public sectors and receives funding from some of its industry partners. It has organisational firewalls to protect it against any undue influences in its work or the publication of its findings. All industry partnerships are subject to review by an independent scientific advisory committee or another independent review body, based on due diligence, TPPs and public sector requirements. FIND catalyses product development, leads evaluations, takes positions and accelerates access to tools identified as serving its mission. It provides indirect support to industry (e.g. access to open specimen banks, a clinical trial platform, technical support, expertise, laboratory capacity strengthening in LMICs) to facilitate the development and use of products in these areas. FIND also supports the evaluation of prioritised assays and the early stages of implementation of WHO-approved (guidance and $\mathrm{PQ}$ ) assays using donor grants. In order to carry out test validations and evaluations, FIND has product evaluation agreements with several private sector companies for the diseases FIND works in which strictly define its independence and neutrality vis-à-vis the companies whose products get evaluated, and describes roles and responsibilities. M. Ruhwald has nothing to disclose.

Support statement: This work was funded by the Global Health Innovative Technology (GHIT) Fund (G2017-201) and the German Federal Ministry of Education and Research through Kreditanstalt fur Wiederaufbau (KfW) (2020 60 457). A.D. Kerkhoff received funding from the National Institute of Allergy and Infectious Diseases (T32 Al060530). The funders had no role in study design, data collection and analysis, decision to publish or preparation of the manuscript. Funding information for this article has been deposited with the Crossref Funder Registry.

\section{References}

$1 \quad$ World Health Organization. High priority target product profiles for new tuberculosis diagnostics: report of a consensus meeting. 2014. www.who.int/tb/publications/tpp_report/en Date last accessed: 24 April 2021.

2 World Health Organization. Lateral flow urine lipoarabinomannan assay (LF-LAM) for the diagnosis of active tuberculosis in people living with HIV: policy update 2019. 2019. www.who.int/tb/publications/2019/ LAMPolicyUpdate2019/en Date last accessed: 24 April 2021.

3 Hunter SW, Gaylord H, Brennan P. Structure and antigenicity of the phosphorylated lipopolysaccharide antigens from the leprosy and tubercle bacilli. J Biol Chem 1986; 261: 12345-12351.

4 Bulterys MA, Wagner B, Redard-Jacot M, et al. Point-of-care urine LAM tests for tuberculosis diagnosis: a status update. J Clin Med 2020; 9: 111.

5 Singhroy DN, MacLean E, Kohli M, et al. Adoption and uptake of the lateral flow urine LAM test in countries with high tuberculosis and HIV/AIDS burden: current landscape and barriers. Gates Open Res 2020; 4: 24.

6 Bjerrum S, Schiller I, Dendukuri N, et al. Lateral flow urine lipoarabinomannan assay for detecting active tuberculosis in people living with HIV. Cochrane Database Syst Rev 2019; 10: CD011420.

7 Broger T, Nicol MP, Székely R, et al. Diagnostic accuracy of a novel tuberculosis point-of-care urine lipoarabinomannan assay for people living with HIV: a meta-analysis of individual in- and outpatient data. PLoS Med 2020; 17: e1003113.

8 Broger T, Nicol M, Sigal G, et al. Diagnostic accuracy of three urine lipoarabinomannan tuberculosis assays in HIV-negative outpatients. J Clin Invest 2020; 130: 5756-5764.

9 Broger T, Sossen B, du Toit E, et al. Novel lipoarabinomannan point-of-care tuberculosis test for people with HIV: a diagnostic accuracy study. Lancet Infect Dis 2019; 19: 852-861.

10 Kandhakumari G, Stephen S. Evaluation of a new rapid kit, BD MGIT TBc identification test for confirmation of Mycobacterium tuberculosis complex. Ind J Pathol Microbiol 2017; 60: 243-246.

11 Foundation for Innovative New Diagnostics. Fujifilm SILVAMP TB LAM test procedure. 2019. https://youtu.be/ aK-QtzkLBug Date last accessed: 24 April 2021. 
12 Broger $T$, Muyoyeta $M$, Kerkhoff $A D$, et al. Tuberculosis test results using fresh versus biobanked urine samples with FujiLAM. Lancet Infect Dis 2020; 20: 22-23.

13 Dorman SE, Schumacher SG, Alland D, et al. Xpert MTB/RIF Ultra for detection of Mycobacterium tuberculosis and rifampicin resistance: a prospective multicentre diagnostic accuracy study. Lancet Infect Dis 2018; 18: 76-84.

14 Albert H, Nathavitharana RR, Isaacs C, et al. Development, roll-out and impact of Xpert MTB/RIF for tuberculosis: what lessons have we learnt and how can we do better? Eur Respir J 2016; 48: 516-525.

15 Kerkhoff AD, Sossen B, Schutz C, et al. Diagnostic sensitivity of SILVAMP TB-LAM (FujiLAM) point-of-care urine assay for extra-pulmonary tuberculosis in people living with HIV. Eur Respir J 2020; 55: 1901259.

16 Sigal GB, Pinter A, Lowary TL, et al. A novel sensitive immunoassay targeting the 5-methylthio-D-xylofuranoselipoarabinomannan epitope meets the WHO's performance target for tuberculosis diagnosis. $J$ Clin Microbiol 2018; 56: e01338-18. 